\title{
Rare vascular anomaly of the common hepatic artery in gastrectomy
}

Keisuke Noda ${ }^{1}$, Shinichiro Kobayashi ${ }^{1}$, Mampei Yamashita ${ }^{1}$, Takayuki Tokunaga ${ }^{1}$, Takanori Hirayama ${ }^{1}$, Koji Natsuda ${ }^{1}$, Kosho Yamanouchi ${ }^{1}$, Hiroaki Takeshita ${ }^{1}$, Tamotsu Kuroki $^{1}$, and Shigeto Maeda ${ }^{1}$

${ }^{1}$ National Hospital Organisation Nagasaki Medical Center

June 28, 2020

\begin{abstract}
Aberrant common hepatic artery originating from the left gastric artery without connecting the gastroduodenal artery is extremely rare. In gastrectomy, accidental ligation or injury of the aberrant common hepatic artery can lead to hepatic arterial ischemia. Reconstructions of CT images may be useful to avoid complications related to vascular anomaly.
\end{abstract}

\section{Clinical Image}

A 63-year-old man presented with advanced gastric cancer. Chemotherapy followed by distal gastrectomy with D2 lymph node dissection was planned. Multiplanar reconstruction in computed tomography (CT) findings showed a vascular anomaly of the celiac axis (CA): the common hepatic artery (CHA) and the proper hepatic artery were absent around the portal vein and, moreover, an aberrant CHA originated from the left gastric artery (LGA) (Fig. 1). Three-dimensional CT angiography revealed that there was no perfusion of the liver from the gastroduodenal artery (GDA) (Fig. 2). Intraoperative findings also showed an aberrant CHA originating from the LGA (Fig. 3). The aberrant CHA was preserved, and the distal side of the LGA was resected to prevent hepatic arterial ischemia. Song's and Adachi's studies of the classifications of CA variations demonstrated that CHA, which was absent in the suprapancreatic area, was in $2 \%$ of cases $(1,2)$. In addition, an aberrant CHA originating from the LGA was demonstrated in $0.16 \%$ of cases (2 ). However, there were no cases of aberrant CHA originating from the LGA without connecting the GDA. Observation of the vascular anatomy around the stomach using a CT reconstruction system may be useful to avoid complications related to vascular anomaly.

\section{Disclosure and Competing Interests}

The authors declare no conflicts of interest in association with the present study.

\section{Consent for publication}

Informed consent was obtained from the patient in this report.

\section{References}

1. Adachi B, Hasebe K, Kyoto Teikoku D, Kenkyusha, History of Medicine C, Paul Kligfield C. Das Arteriensystem der Japaner1928.

2. Song SY, Chung JW, Yin YH, Jae HJ, Kim HC, Jeon UB, et al. Celiac axis and common hepatic artery variations in 5002 patients: systematic analysis with spiral CT and DSA. Radiology. 2010;255(1):278-88.

Figure Legends 


\section{Figure 1.}

Multiplanar reconstruction in CT examination demonstrates that CHA and the proper hepatic artery are absent around the PV (triangle) and that an aberrant CHA (arrow) originates from the LGA. CHA, common hepatic artery; PV, portal vein.

Multiplanar reconstruction in CT angiography also demonstrates that aberrant CHA originates from LGA and that there is no perfusion of the liver from GDA. \#1; LGA (left gastric artery), \#2; aberrant CHA (common hepatic artery), \#3; GDA (gastroduodenal artery), \#4; PV (portal vein)

\section{Figure 2.}

$\mathrm{CT}$ angiographic reconstruction using volume rendering. Reconstructed 3D image clearly shows an aberrant CHA, which is a branch from the LGA. The GDA originating from the SMA is completely separated from the CHA. \#1; celiac axis, \#2; LGA (left gastric artery), \#3; aberrant CHA (common hepatic artery), \#4; splenic artery, \#5; SMA (superior mesenteric artery), \#6; GDA (gastroduodenal artery)

\section{Figure 3.}

Intraoperative image of aberrant CHA and LGA. The CHA is absent in the suprapancreatic area. An aberrant CHA (triangle) originating from the LGA (arrow) is present. CHA, common hepatic artery; LGA, left gastric artery.

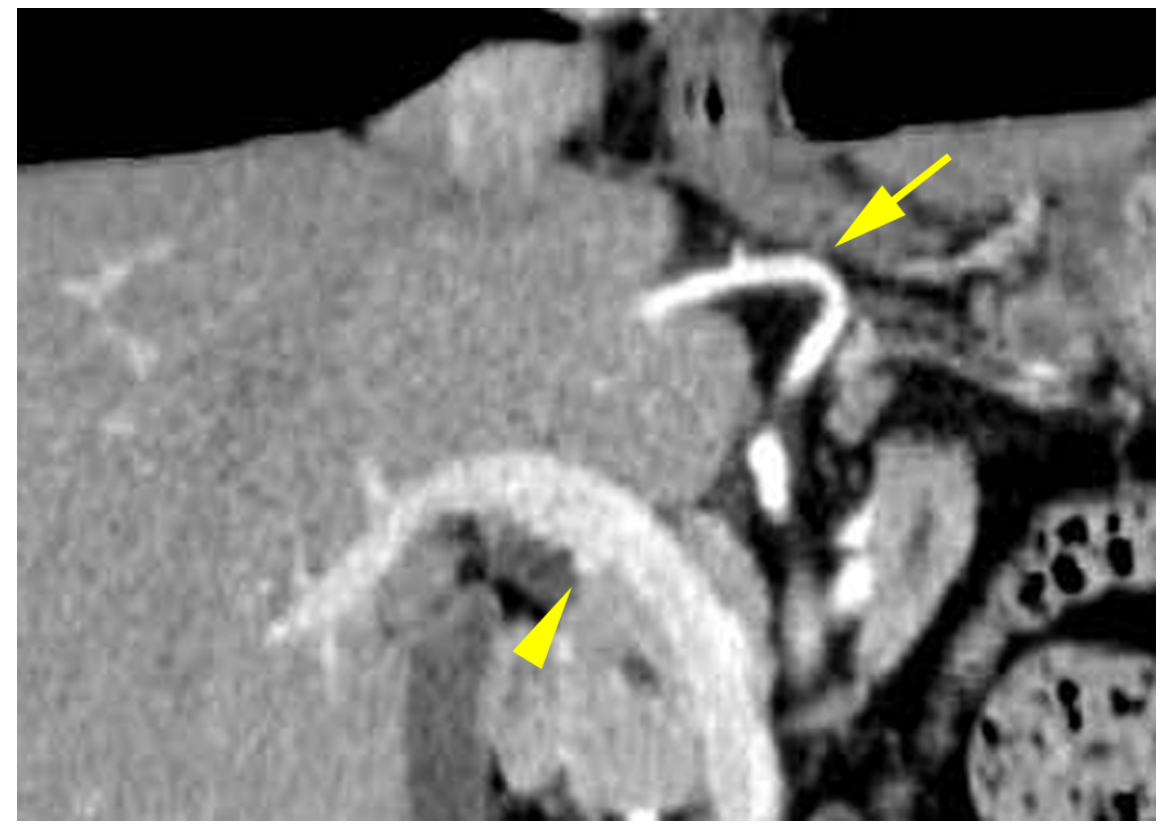



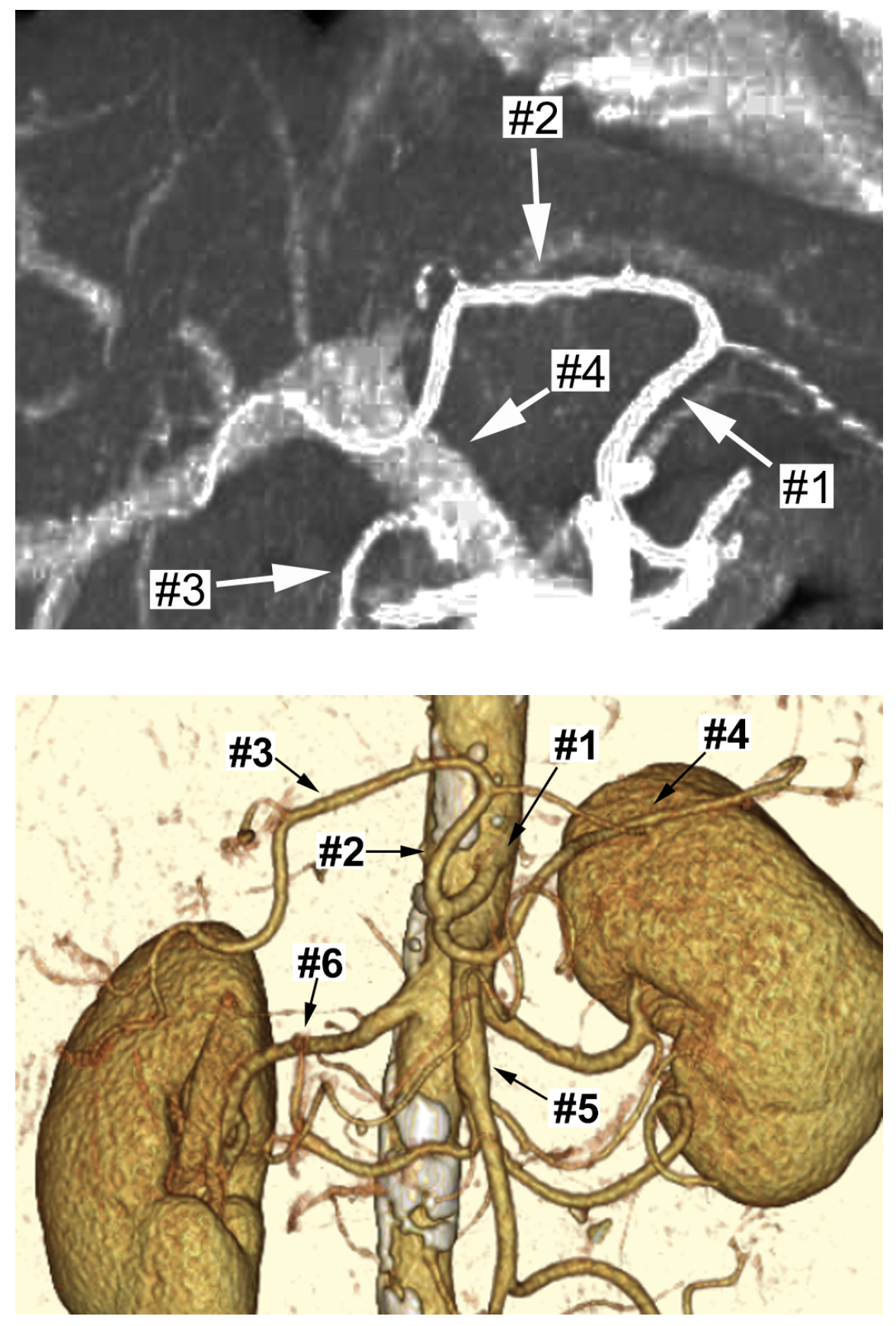


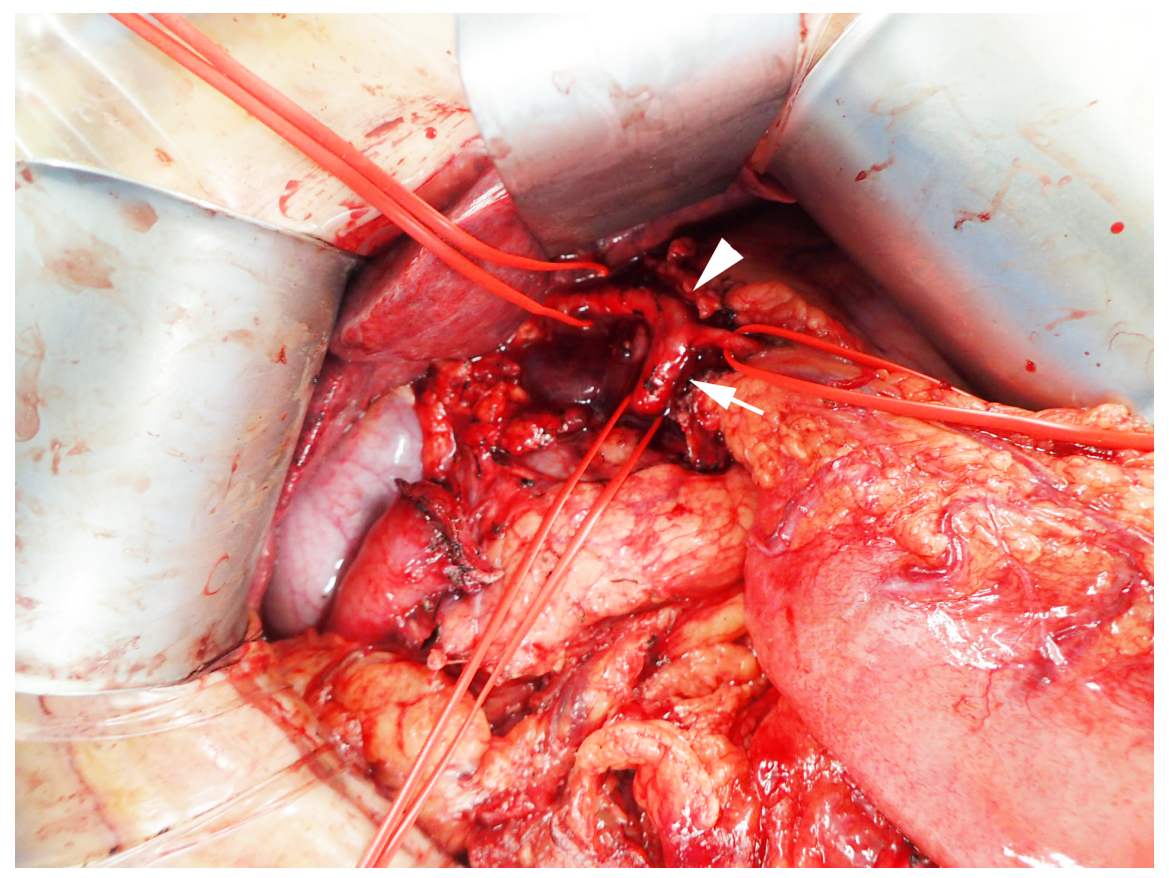

